Cahiers $d u$ MONDE RUSSE

\section{Cahiers du monde russe}

Russie - Empire russe - Union soviétique et États indépendants

$49 / 4 \mid 2008$

Destins individuels et terreur. Jeunesse dans la société post-stalinienne

\title{
Alena V. Ledeneva, How Russia Really Works
}

\section{Andreï Kozovoï}

\section{(2) OpenEdition}

Journals

Édition électronique

URL : https://journals.openedition.org/monderusse/6976

DOl : $10.4000 /$ monderusse. 6976

ISSN : $1777-5388$

Éditeur

Éditions de l'EHESS

Édition imprimée

Date de publication : 28 décembre 2008

Pagination : 795-797

ISBN : 978-2-7132-2197-2

ISSN : $1252-6576$

Référence électronique

Andreï Kozovoï, « Alena V. Ledeneva, How Russia Really Works », Cahiers du monde russe [En ligne], 49/4 | 2008, mis en ligne le 24 décembre 2009, consulté le 04 septembre 2022. URL : http://

journals.openedition.org/monderusse/6976 ; DOI : https://doi.org/10.4000/monderusse.6976

Ce document a été généré automatiquement le 4 septembre 2022

Tous droits réservés 


\title{
Alena V. Ledeneva, How Russia Really Works
}

\author{
Andreï Kozovoï
}

\section{RÉFÉRENCE}

Alena V. LEDENEVA, How Russia Really Works. The Informal Practices That Shaped

post-Soviet Politics and Business. Ithaca-Londres : Cornell University Press, 2006, 270 p. (Culture \& Society after Socialism).

1 Après s'être penchée sur le blat (réseau de faveurs) à l'époque soviétique ${ }^{1}$, Alena Ledeneva, lectrice à l'University College de Londres, poursuit son enquête sur les pratiques informelles dans les milieux politiques et d'affaires, cette fois dans la Russie des années 1990. Fruit de huit années de travail, son ouvrage, au croisement de l'ethnologie et de la science politique, est nourri de soixante-deux interviews menées entre 1997 et 2003.

2 La situation politique et économique russe dans les années 1990 porte l'empreinte de la « thérapie de choc » qui entraîne les Russes dans un tourbillon marqué par la nécessité de faire appel aux « règles non écrites » pour pallier les défauts législatifs et institutionnels de la jeune Fédération de Russie. L'auteur préfère cependant parler de "pratiques informelles ", ensemble de savoir-faire évoluant dans un espace où pratiques formelles et normes informelles interagissent mutuellement. En sept chapitres, Ledeneva décrit ces nouvelles pratiques nées avec l'introduction de la démocratie en Russie, en recourant à de très nombreux exemples; puis, elle s'emploie à les resituer dans la gouvernance russe. Cherchant à dépasser les clichés immémoriaux sur l'incapacité russe à instaurer une véritable démocratie, le mépris traditionnel de la loi ou la kleptomanie naturelle des habitants..., l'auteur brosse le tableau d'une politique et d'une économie russes largement dépendantes de ces pratiques informelles, mais elle montre également ces dernières contribuant, paradoxalement, à renforcer en retour politique et économie. Ainsi, à propos de la fameuse question des fuites de capitaux, elle invoque l'exemple de 
Chypre - à la fois zone franche favorite pour les expatriations et source d'investissements en Russie comparable aux capitaux français et britanniques (ch. 1).

Ledeneva applique d'abord le concept de pratiques informelles au champ politique. L'apparition des élections libres dans la Russie des années 1990 et un ensemble de facteurs, dont la relative " malléabilité du public » russe d'alors, permettent l'émergence d'une pratique nouvelle (aux racines cependant anciennes), le černyj piar ${ }^{2}$, qui consiste à affaiblir un adversaire politique, ou économique, dans les médias, et à aller parfois jusqu'à ruiner sa réputation, en utilisant la capacité de ces derniers à se vendre au plus offrant. L'auteur construit une typologie des pratiques de ce piar, qui va du spectre de la légalité à l'illégalité, mais y intègre également - et c'est là toute son originalité - la notion d'éthique, ce qui lui permet de parler de seryj piar (relations publiques " grises ») pour des pratiques illégales mais moralement justifiées et des pratiques légales mais immorales. Dans tous les cas, le černyj piar apparait comme une technique électorale souvent à la limite de l'illégalité, qui profite des contradictions législatives mais rend en même temps le processus démocratique possible (ch. 2).

4 Ledeneva s'attarde ensuite sur le kompromat (dossier compromettant), pratique héritée de l'époque soviétique (ch. 3). Le terme a été popularisé en Occident après que le ministre de la Justice Valentin Kovalev a été photographié avec des prostituées en 1997 (lui ou son sosie...), ce qui lui coûta son poste. Fruit et manifestation la plus éclatante de la liberté d'expression, le kompromat n'aboutit pas la plupart du temps à un renforcement de la démocratie russe, comme dans les anciens pays communistes est-européens, où cette pratique avait permis de mettre fin à la carrière d'apparatchiks compromis avec les crimes de l'ancien régime. Le kompromat, mais aussi la krugovaja poruka (cercle consensuel), qui remonte quant à elle à l'époque tsariste (ch.4), sont des pratiques complémentaires du černyj piar. Figurant une situation dans laquelle tous les membres d'un groupe donné sont liés, et responsables les uns devant les autres, la krugovaja poruka se retrouve à tous les niveaux de la société, tant dans les sphères culturelle qu'économique et politique (où elle fait écho à la notion de " clan »).

Dans les trois chapitres suivants, l'auteur étudie les pratiques informelles liées aux milieux économiques et financiers, comme le «troc parallèle» (échanges interentreprises, tenevoj barter, ch.5), les techniques de la « comptabilité occulte » (dvojnaja bukhgalterija, ch. 6) et le milieu et les pratiques des « pousseurs d'affaires» (tolkači, ch. 7), dont les fameux « toits » (kryšy) pour les entreprises menacées (y compris physiquement) par des concurrents. L'objectif est de montrer que, contrairement à ce que prétendent ses dirigeants, la Russie n'est ni une démocratie, ni un pays à économie de marché comparable à ses modèles occidentaux reconnus. On reste interdit devant l'ingéniosité déployée par les hommes d'affaires russes pour survivre face à des contraintes structurelles (règles du jeu économique imposées d'en haut) et conjoncturelles (contexte de transition chaotique et de système bancaire déficient), et contourner le système tout en contribuant à sa survie, en particulier dans le cadre de la crise de 1998.

Dans la lignée d'un Alexei Yurchak ou d'une Caroline Humphrey³ , l'ouvrage de Ledeneva cherche à analyser la part de la culture publique russe qui se trouve habituellement dans les journaux à sensation ou dans les discours des acteurs du secteur informel. Chemin faisant, l'auteur réussit son pari de transcender les grilles d'analyse traditionnelles. Bien qu'elle conserve l'opposition entre domaines formel et informel, elle replace le dernier dans une relation dynamique; dès lors les pratiques informelles apparaissent dans toute 
leur ambivalence, ce qui interdit les simplifications que l'on trouve encore trop fréquemment dans les manuels sur la période ${ }^{4}$.

7 On regrettera cependant que, pour échapper au «déterminisme ethnique " (voir les clichés énumérés ci-dessus), l'auteur verse dans un « déterminisme systémique », l'état de la Russie dans les années 1990 ne laissant à sa population (dans son entier, et pas seulement ses éléments criminels) d'autre choix que de faire siennes les pratiques informelles - ce qui revient d'une certaine manière à les banaliser. Au fond, en s'appuyant exclusivement sur les interviews des acteurs de l'économie informelle, Ledeneva donne l'impression qu'elle leur est finalement favorable. De fait, elle montre indirectement ce que le film L'oligarque avait donné en son temps à voir au grand public $^{5}$ : que les persécutions des « oligarques» russes sont juridiquement infondées et demeurent donc un fait du Prince. Parfois, le travail manque de perspective géographique et historique. Le parallèle avec les pratiques informelles d'autres pays n'est qu'effleuré. De même, l'auteur fait simplement allusion à la question pourtant centrale des relations entre société et loi dans l'histoire longue de la Russie pour expliquer la popularité des pratiques informelles. Enfin, des citations-illustrations et l'emploi d'un vocabulaire emprunté à des autorités (contestables), tel Pierre Bourdieu, se substituent parfois à une réflexion personnelle sans que cela soit vraiment justifié ${ }^{\text {. }}$

En dépit de ces réserves, le travail d'Alena Ledeneva reste particulièrement stimulant pour définir la fameuse "zone grise » dans laquelle se trouve la Russie dans les années 1990, constater que l'héritage soviétique est toujours présent malgré la répudiation du régime, et saisir mieux, enfin, les origines de la popularité de Putin.

\section{NOTES}

1. Russia's Economy of Favours, Cambridge, MA : Cambridge University Press, 1998.

2. Expression difficile à traduire : si le russe est un calque de l'anglais «black $P R$ », le français est moins souple. « Relations publiques (RP) noires ", " marketing noir » sont des pis-aller.

3. Alexei Yurchak, Everything was Forever, until it was no more. The Last Soviet Generation, Princeton : Princeton University Press, 2005 ; Caroline Humphrey, The Un-making of Soviet Life. Everyday Economies after Socialism, Ithaca, Cornell University Press, 2002.

4. Voir par exemple l'ouvrage dirigé par Marie-Pierre Rey, Les Russes de Gorbatchev à Poutine, Paris, Armand Colin, 2005.

5. Réalisé par Pavel Lungin en 2002. Mal traduit en France par Un Nouveau Russe, le film raconte l'épopée de Platon Makovskij, personnage inspiré de la carrière de Boris Berezovskij.

6. Il est également regrettable que l'auteur n'ait pas cherché à traduire «Le lièvre et le renard : un conte d'aujourd'hui », une savoureuse illustration du chapitre sur les tolkači (p. 165). 